\title{
Dynamic Monitoring of Vegetation Coverage by Remote Sensing in Longnan City of Gansu Province based on VBSI
}

\author{
Songlin Zhang ${ }^{a}$, Jinxiu Wang ${ }^{b}$ and Shuzhi $\mathrm{Ji}^{\mathrm{c}}$ \\ College of Geography \& Environment Science, Northwest Normal University, Lanzhou 730070, PR \\ China \\ a13893314212@163.com, nwnujinxiu_w@163.com, nwnujsz@163.com
}

\begin{abstract}
Keywords: vegetation coverage; VBSI; mixed pixel decomposition model; remote sensing monitoring.
\end{abstract}

Abstract. Vegetation coverage is an important index to measure ecological environment. In this paper, the composite vegetation index (VBSI), which can weaken the interference of mountain shadow, soil background, rocks and buildings, was constructed. Combined with mixed pixel decomposition model, the dynamic monitoring of vegetation coverage in Longnan city in 1995 and 2015 was carried out. The results showed that the interference of shading information using VBSI for vegetation coverage estimation can be reduced to $45.77 \%$ of NDVI. Dynamic monitoring showed that the vegetation coverage in Longnan city had an increasing trend. The areas of low vegetation coverage decreased slightly whereas the areas of medium and high vegetation coverage increased significantly. High vegetation coverage areas were mainly distributed in Huixian county, Chengxian county, Liangdang county, Wudu district and the vicinity of Bailongjiang river where the project of conversion of degraded farmland into forest has implemented efficiently in Longnan city.

\section{Introduction}

Vegetation coverage is effected by regional climate, environment and other factors. Vegetation coverage variation is a significant factor in evaluation of regional ecosystem [1]. Vegetation coverage refers to the percentage of vegetation vertical projection area on the ground, which is a comprehensive quantitative index of vegetation coverage [2]. With the development of remote sensing technology, the method of estimating vegetation coverage has progressed from the traditional field measurement method to the remote sensing data method, which provides the possibility for vegetation coverage monitoring in the large-scale regions [3]. Remote sensing method of measuring vegetation coverage mainly includes: regression model method [4], vegetation coverage [5] and pixel decomposition model method [6]. However, the regression model method has certain limitations in that it is only applicable to specific areas and vegetation types, and relies heavily on the measured data to establish the model. Many studies have demonstrated that the models set up by vegetation index method to estimate vegetation coverage can form a general calculation method [7, 8]. Normalized difference vegetation index (NDVI) [9], ratio vegetation index (RVI) [10], modified vegetation index (MVI) [11], and soil regulated vegetation index (SAVI) [12] are commonly used.

At present, normalized vegetation index (NDVI) is one of the most widely used vegetation indexes [13]. However, NDVI has a relatively higher deviation in assessment of high density vegetation area due to the influence of shadow [14]. Inspired by the forest canopy density (FCD) model principle, the composite vegetation index (VBSI), which can weaken the influence of mountain shadow, soil background, rocks and buildings is constructed in consideration of the geomorphological characteristics of Longnan city. VBSI is compared with NDVI in terms of weakening effect of shadow interference. Based on mixed image element model, the vegetation coverage and change of Longnan city are estimated by VBSI. It will be conducive to understanding the distribution of vegetation in Longnan city from the spatial and temporal dimension. Moreover, it can provide a scientific basis for promoting vegetation restoration and reconstruction in this area. 


\section{Overview of study area}

Longnan city is located in the southeast of Gansu Province $\left(104^{\circ} 01^{\prime}-106^{\circ} 34^{\prime} \mathrm{E} 、 32^{\circ} 35^{\prime}-34^{\circ} 32^{\prime} \mathrm{N}\right)$. It lies on the north of Qinling Mountains, south of Sichuan Basin, west of Gannan Plateau, east of Qinba Mountain. Longnan city governs one district, Wudu district, Tanchangxian country, Chengxian country, Kangxian country, Wenxian country, Xihexian countryn, Lixian country, Huixian country, covering a total area of $27.90 \mathrm{~km}^{2}$. The location map of Longnan city is shown in Fig. 1.

Climate in Longnan city is complex and diverse due to the influence of geographical location, topography, atmospheric environment, solar radiation. Annual average temperature is 5-16 $\square$. Annual precipitation is $400-1000 \mathrm{~mm}$. Longnan city is the transition zone of hot humid, warm, humid and semi humid plateau varied climate where exits a number of rare plants and animals [15]. The main topography type is mountain and hills in Longnan city which is the major area for implementing the project of conversion of degraded farm land into forest [16]. Therefore, the study on dynamic coverage of vegetation coverage in Longnan has important theoretical and practical value.

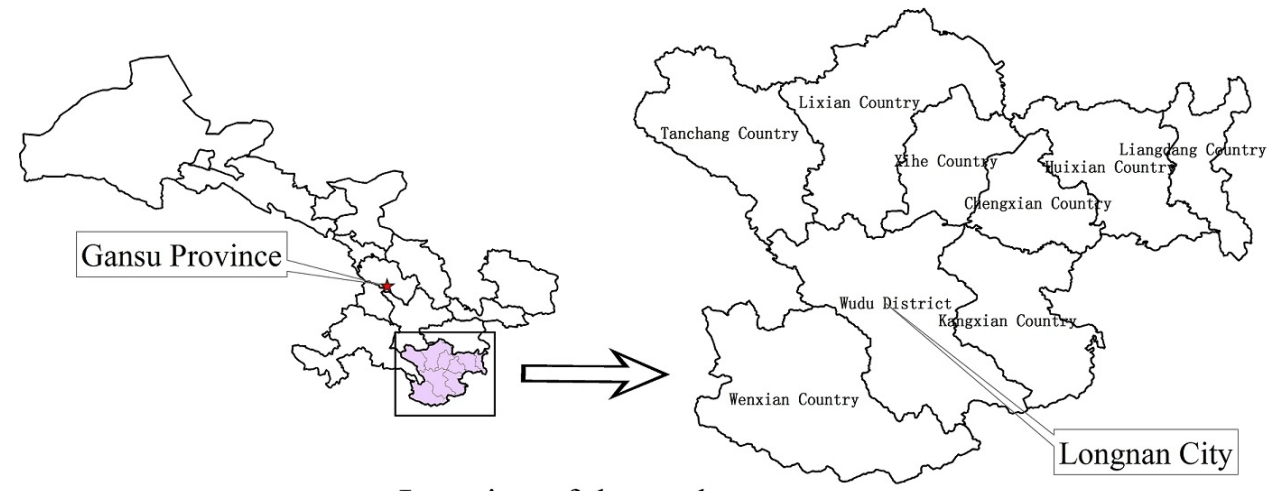

Location of the study area

Fig. 1 Location map of Longnan city

\section{Data resource and research method}

Data resource. The data were obtained from Landsat TM data $(30 \mathrm{~m} \times 30 \mathrm{~m})$. The two periods imaging time was selected in June, 1995 and 2015, respectively. The vigorous periods of vegetation activity can represent the vegetation growth condition and land use situation in this area, which have the significance of vegetation dynamic monitoring.

In order to ensure the integrity and accuracy of the data, atmospheric correction was carried out for two periods images.

VBSI vegetation index. The composite vegetation index, VBSI is constructed on linear combination of three indexes, vegetation index $(V I)$, bare soil index $(B I)$, shadow index $(S I)$. The basic formula is $[17,18]$ :

$$
V B S I=f(V I, B I, S I)=V I+n \times B I+S I
$$

Where $n$ is the correction coefficient determined by actual situations and experiments, taking $n$ -0.1 .

According to the FCD model (forest canopy density model) proposed by the ITTO (International Tropical Timber Organization), the vegetation index $(V I)$, the bare soil index $(B I)$ and the shading index $(S I)$ are constructed by the green band $\left(B_{g}\right)$, red band $\left(B_{r}\right)$, near infrared band $\left(B_{i r}\right)$ and short wave infrared band $\left(B_{s r}\right)$ (using NDVI for VI) [19, 20]:

$$
\begin{aligned}
& V I=N D V I=\left(B_{i r}-B_{r}\right) /\left(B_{i r}+B_{r}\right) \\
& B I=\left(B_{s r}+B_{r}-B_{i r}\right) /\left(B_{s r}+B_{r}+B_{i r}\right)
\end{aligned}
$$




$$
S I=\sqrt{\left(256-B_{g}\right)\left(256-B_{r}\right)}
$$

Mixed pixel decomposition model. A pixel in a remote sensing image may actually contain multiple parts, each of which contributes to the remote sensing information. Therefore, the remote sensing information (band or vegetation index) can be decomposed to create a pixel decomposition model for estimation of vegetation fraction [21].

The mixed pixel decomposition method assumes that the information $\mathrm{S}$ of a remote sensing image pixel can be divided into vegetation information $S_{\text {veg }}$ and non-vegetation information $S_{\text {soil }}$. The vegetation coverage fraction is $f_{c}$. Therefore, pixel information $S$ of the remote sensing image may be expressed as [22]:

$$
S=f_{c} \times S_{\text {veg }}+\left(1-f_{c}\right) \times S_{\text {soil }}
$$

The vegetation coverage of the formula through the deformation for the above formula can be expressed as:

$$
f_{c}=\left(S-S_{\text {soil }}\right) /\left(S_{\text {veg }}-S_{\text {soil }}\right)
$$

The mixed pixel decomposition model reflects the relationship between remote sensing information and vegetation coverage. It can weaken the influence of air, soil background and vegetation type, preserving the information of vegetation coverage better [17].

\section{Result and discussion}

Comparison between VBSI and NDVI. In order to verify the effect of VBSI, a comparison was made between VBSI and NDVI in reducing the influence of mountain shadow. The VBSI and NDVI vegetation index values were extracted from TM images in 2015 of eight counties and one district of the sunny and shady slope of the adjacent hill. The relative deviation of the vegetation index of sunny slopes is: (vegetation index value of sunny slope - vegetation index value of shady slope) / vegetation index value of sunny slope. The relative deviation ratio = relative deviation of NDVI / relative

\begin{tabular}{|c|c|c|c|c|c|c|c|c|c|}
\hline Sample & 1 & 2 & 3 & 4 & 5 & 6 & 7 & 8 & 9 \\
\hline \multirow{2}{*}{ ongitude and latitude } & $105^{\circ} 1^{\prime} \mathrm{E}$ & $104^{\circ} 18^{\prime} \mathrm{E}$ & $05^{\circ} 34^{\prime} \mathrm{E}$ & $05^{\circ} 35^{\prime} \mathrm{E}$ & $04^{\circ} 36^{\prime} \mathrm{E}$ & $05^{\circ} 19^{\prime} \mathrm{E}$ & $05^{\circ} 5^{\prime} \mathrm{E}$ & $05^{\circ} 54^{\prime} \mathrm{E}$ & $106^{\circ} 28^{\prime} \mathrm{E}$ \\
\hline & $33^{\circ} 21^{\prime} \mathrm{N}$ & $33^{\circ} 6^{\prime} \mathrm{N}$ & $3^{\circ} 45^{\prime} \mathrm{N}$ & $3^{\circ} 16^{\prime} \mathrm{N}$ & $: 2^{\circ} 49^{\prime} \mathrm{N}$ & $i 3^{\circ} 59^{\prime} \mathrm{N}$ & $4^{\circ} 21^{\prime} \mathrm{N}$ & $3^{\circ} 59^{\prime} \mathrm{N}$ & $33^{\circ} 40^{\prime} \mathrm{N}$ \\
\hline NDVI in sunny slope & 0.595 & 0.634 & 0.661 & 0.696 & 0.675 & 0.436 & 0.447 & 0.704 & 0.791 \\
\hline NDVI in shady slope & 0.486 & 0.455 & 0.537 & 0.528 & 0.601 & 0.381 & 0.394 & 0.529 & 0.663 \\
\hline $\begin{array}{c}\text { ative deviation of NDVI } \\
1 \%\end{array}$ & 18.32 & 28.23 & 18.76 & 24.14 & 10.96 & 12.61 & 11.86 & 24.86 & 16.18 \\
\hline VBSI in sunny slope & 0.662 & 0.707 & 0.726 & 0.754 & 0.747 & 0.537 & 542 & 0.762 & 0.856 \\
\hline VBSI in shady slope & 0.564 & 0.604 & 0.663 & 0.638 & 0.709 & 0.506 & 0.518 & 0.662 & 0.786 \\
\hline tive deviation of VBSI / \% & 14.80 & 14.57 & 8.68 & 15.38 & 5.09 & 5.77 & 4.43 & 13.12 & 8.18 \\
\hline relative deviation ratio & 1.24 & 1.94 & 2.16 & 1.57 & 2.16 & 2.19 & 2.68 & 1.89 & 1.98 \\
\hline
\end{tabular}
deviation of VBSI $\times 100 \%$. The results are shown in Table 1 .

Table 1 Comparison between VBSI and NDVI value in reducing interference shadow of the images 
Due to different climatic topography in Longnan city, the sample sites (1-9) were selected in Wudu district, Tanchang country, Chengxian country, Kangxian country, Wenxian country, Xihexia countryn, Lixian country, Huixian country, respectively.

From Table 1, the relative deviations of the average VBSI and NDVI vegetation index were $18.44 \%$ and $10.00 \%$, respectively. The average relative deviation of NDVI was about 1.8 times the value of VBSI. When vegetation coverage was estimated by VBSI, the interference effect of image shadow can be cut to $45.77 \%$ of NDVI, which indicated that VBSI was more suitable for vegetation coverage evaluation in the study area than NDVI.

Vegetation coverage in Longnan city. Based on the above analysis, combined with mixed pixel model, VBSI was used to estimate the vegetation in Longnan city in June 1995 and 2015. In order to express the distribution of vegetation coverage intuitively, according to the Classification Criteria of Soil Erosion (SL.190-96) promulgated by the Ministry of Water Resources, combined with the specific situation of Longnan city, the vegetation coverage was divided into five levels: no vegetation $(0 \%)$, low vegetation coverage $(0<\mathrm{fc}<15 \%)$, medium vegetation coverage $(15 \% \leq \mathrm{fc}<40 \%)$, upper vegetation coverage $(40 \% \leq \mathrm{fc}<70 \%)$, high vegetation coverage (fc $>70 \%)$ : Vegetation coverage grading results for 1995 and 2015 are shown in Fig. 2.

It can be seen from the Fig. 2 that the high vegetation coverage was mainly distributed in the eastern and southern areas of Longnan city and the low and medium coverage in the northern and western areas. High vegetation coverage areas were mainly distributed in Huixian county, Chengxian county, Liangdang county, Wudu district and the vicinity of Bailongjiang River where the project of conversion of degraded farmland into forest has implemented efficiently in Longnan city.

In addition, the annual precipitation data of Longnan city in 2015 was higher than that in 1995. Therefore, the influence factors of vegetation coverage change in Longnan city can be considered as the influence of climatic factors and the project of the conversion of degraded farm land into forest. The results were in accordance with the estimation of vegetation coverage using NDVI by Wang Chuansheng et al. [23].

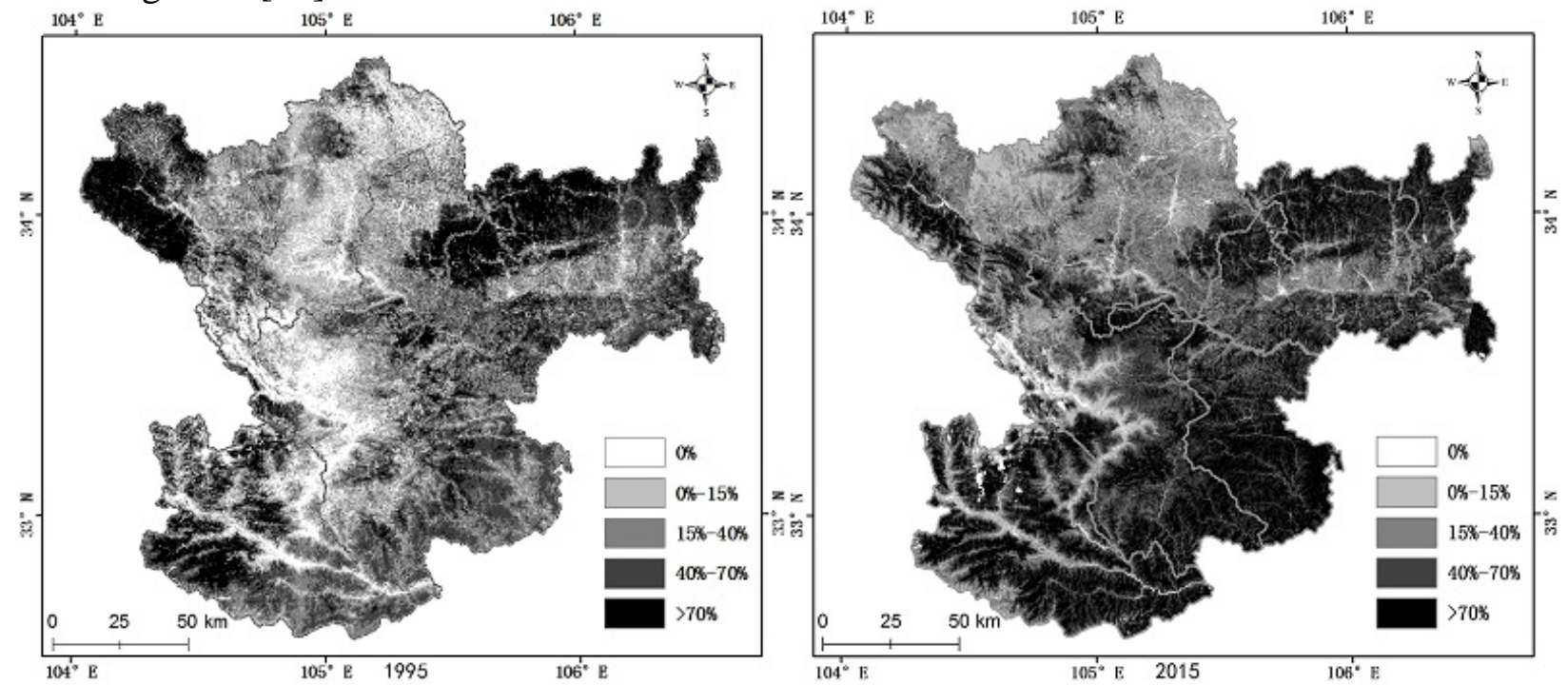

Fig.2 vegetation coverage classification map of Longnan city in 1995 and 2015

The vegetation coverage at all levels was statistically calculated in the two periods. According to the results shown in Fig. 3, low, medium and upper vegetation coverage dominated in 1995, accounting for $21.79 \%, 25.75 \%$ and $25.59 \%$ of the total area, high vegetation coverage for only $15.37 \%$, no vegetation coverage for $11.49 \%$; In comparison, high vegetation coverage and upper vegetation coverage mainly dominated in 2015 , accounting for $31.02 \%$ and $29.76 \%$, no vegetation coverage for $2.42 \%$, low vegetation coverage for $13.82 \%$, and medium vegetation coverage for $22.98 \%$. It can be seen that no vegetation coverage and low vegetation coverage in Longnan city decreased obviously, the medium vegetation coverage slightly, and the high vegetation coverage 
increased significantly from 1995 to 2015 . The variation indicated that the vegetation in Longnan city has been effectively restored and the vegetation has entered a good growth period in 2015 .

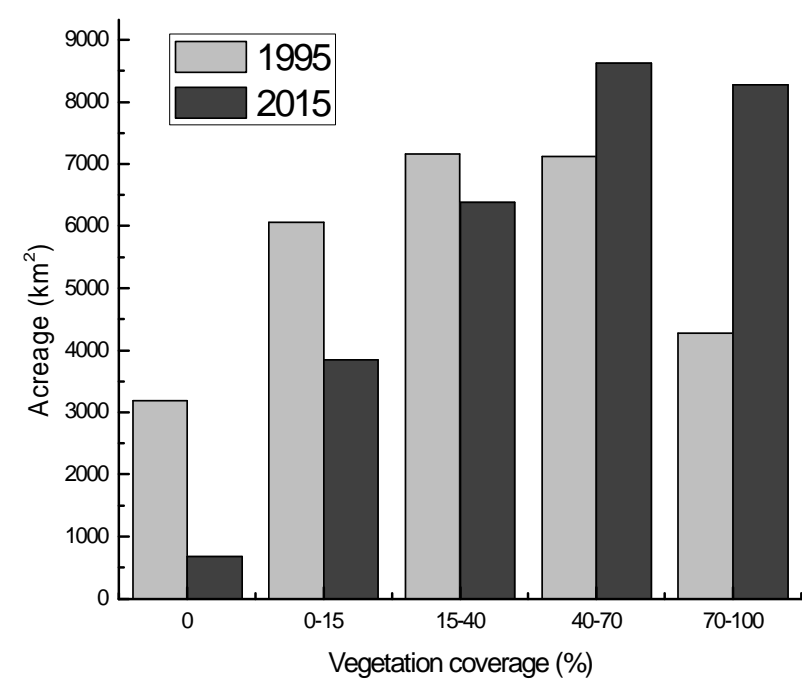

Fig. 3 Comparison of vegetation coverage at different levels in 1995 and 2015

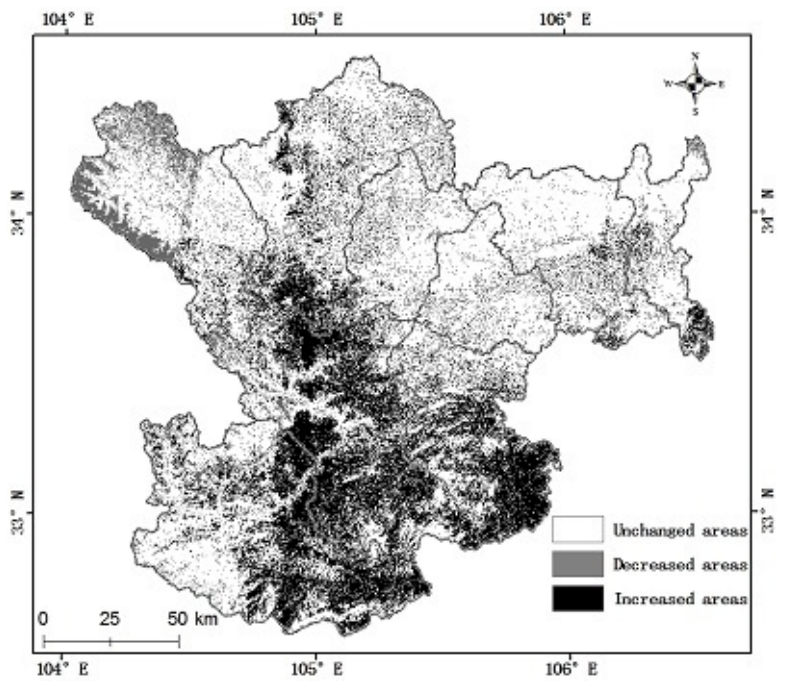

Fig. 4 Dynamic monitoring distribution map of vegetation coverage in Longnan city

Dynamic Monitoring of Vegetation Coverage in Longnan city. The dynamic monitoring results of vegetation coverage in Longnan city from 1995 to 2015 are shown in Fig. 4. Combined with statistical analysis, it can be observed that the unchanged vegetation coverage area covered $18650.60 \mathrm{~km}^{2}$, accounting for $67.07 \%$ of the total area; the increased vegetation coverage area covered $7688.03 \mathrm{~km}^{2}$, accounting for $27.65 \%$ of the total area, showing that the vegetation change in Longnan city showed a stable rise trend. The decreased vegetation coverage area was relatively scattered, covering $1452.44 \mathrm{~km}^{2}$, accounting for $5.22 \%$ of the total area. It mainly resulted from the economic activities, real estate development activities and major transport construction and, such as National Highway, Jiang Wu Road and other 11 provincial trunk road constructions, Lanyu Railway, Lancheng Railway, Chengwu Airport and Chengzhou Airport construction [23].

\section{Conclusions}

Compared with NDVI, VBSI was more suitable for estimating vegetation coverage in the study areas. The interference effect of remote sensing images shadow for vegetation coverage estimated by VBSI can be reduced to $45.77 \%$ of NDVI. Based on the mixed pixel decomposition model, using VBSI to estimate the vegetation coverage in remote sensing dynamic monitoring has application value.

According to the spatial distribution of vegetation coverage, the areas with high vegetation coverage were mainly distributed in the east and the south of Longnan city. The low and medium vegetation coverage areas were mainly distributed in the northern and western region.

According to the time distribution of vegetation coverage, Longnan city was dominated by low and medium vegetation coverage in 1995 while it was mainly dominated by upper and high vegetation coverage in 2015, indicating that vegetation in Longnan city has been effectively restored and entered a better growth period.

The increase of vegetation coverage in Longnan city was mainly due to the implementation of the project of returning farmland to forest. The increase of rainfall was also a favorable factor for it. The economic construction activities were the main reasons for the reduction of vegetation coverage in 
some areas. Hence, the economic construction should be coordinated with the construction of ecological civilization.

\section{Acknowledgements}

The authors are very grateful for the financial support from the National Natural Science Fund of China (51068025), the Natural Science Fund of Gansu Province in China (1308RJZA302), and the Open Research Fund of Oil and Gas Resources Key Laboratory of Gansu Province in China (SZDKFJJ20150606).

\section{References}

[1] Z. Li, Y. Zhang, and Q. Zhu: Geomorphology, Vol. 228 (2015), p. 462.

[2] D. Chu and C. DuoJi: Remote Sens Technol. \& Appl. Vol. 25 (2010), p. 707. (In Chinese)

[3] R.L. Pu, P. Gong and Y. Tian: Int. J. Remote Sens. Vol. 29 (2008), p. 3987.

[4] Z.J. Gu, Z.Y. Zeng and X.Z. Shi: Int. J. Remote Sens. Vol. 30 (2009), p. 2479.

[5] N. Qin, J. Xu and M. Ji: Environ. Manage. Vol. 50 (2012), p. 405.

[6] B.C. Lv, Z. Wang: submitted to Remote Sensing Information (2003).

[7] Y. Song, C. Zhou and W. Zhang: Ecol. Res. Vol. 26 (2011), p. 79.

[8] Z.Y. Ma, T. Shen and J.H. Zhang: Bull. Surv. \& Map. Vol.1 (2007), p. 45. (In Chinese)

[9] Z. Jiang, A.R. Huete and J. Chen: Remote Sens. Environ. Vol. 101 (2006), p. 366.

[10]H. Yan, C.Y. Wang and Z. Niu: J. Remote Sens. Vol. 6 (2002), p. 30-34. (In Chinese)

[11] S. Xu, R. Shen and X. Yang: Remote Sens. Land \& Resour. Vol. 24 (2012), p. 95.

[12]Z. Jiang, A.R. Huete and J. Qi: J. Appl. Remote Sens. Vol. 1 (2007), p. 3398.

[13]A. Huete, K. Didan and T. Miura: Remote Sens. Environ. Vol. 83 (2002), p. 195.

[14]C. Small: Int. J. Remote Sens. Vol. 22 (2001)1305.

[15]H. Lu, M.R. Raupach and T.R. Mcvicar: Remote Sens. Environ. Vol. 86 (2003), p. 1.

[16]H. Chen, S. Zhu and Q. Li: Mountain Res. Vol. 32 (2014), p. 662.(In Chinese)

[17] J. Hong, W. Qinmin and W. Xiaoqin: J. Nat. Resour. Vol. 21 (2006), p. 126. (In Chinese)

[18]Z.J. Luo, X.M. Liu and Y.L. Liu: Transac. CSAE, Vol. 24 (2008), p. 57. (In Chinese)

[19]X.Q. Li, D.F. Sun, F.R. Zhang: J. MT. Res. Vol. 21 (2003), p. 272.

[20]S. Nandy, P.K. Joshi and K.K. Das: J. Indian Society Remote Sens. Vol. 31 (2003), p. 291.

[21]A. Karnieli: Remote Sens. Rev. Vol. 13 (1996), p. 161.

[22] S. Li, W.Z. Li and J.J. Zhou:Geography and Geo-Infor. Sci. Vol. 5 (2007), p.35. (In Chinese)

[23]C. Wang and Q. Li: Res. Soil Water Conserv. Vol. 23 (2016), p. 308. (In Chinese) 